\title{
Observatorios y alineamientos astronómicos en el Tampu Inka de Huánuco Pampa
}

\begin{abstract}
José Luis Pino Matos*
Resumen El sitio arqueológico de "Huánuco Pampa", uno de los tampus Inka mejor diseñados en los Andes Centrales durante la expansión del Tawantinsuyu, presenta evidencias de importantes alineamientos astronómicos asociados a los principales espacios y estructuras. Estos se pueden observar especialmente en la plataforma "Ushnu" y el "Inkawasi"; entre las "tianas" (asientos de piedra), nichos y accesos principales de ambas estructuras con fina albañilería Inka. Estos alineamientos están asociados a los "pasos solares" a través del zenit, anti-zenit, equinoccio y solsticios; salida y puesta de la Luna en sus posiciones extremas y; la salida y puesta de la Cruz del Sur, Alfa y Beta Centaurus. Con estas evidencias argumentamos la importancia del uso de la astronomía Inka en los grandes centros de control provincial.
\end{abstract}

Palabras clave Tawantinsuyu, astronomía Inka, ushnu, organización del espacio, calendarios regionales.

Thstract archaeological site of Huánuco Pampa, one of the best designed Inka tampus in
the Central Andes during the expansion of the Tawantinsuyu Empire, shows evi-
dences of significant astronomical alignments associated to main spaces and buil-
dings. They can be observed at the "Ushnu" platform and the "Inkawasi"; among
the "Tianas" (stone seats), niches and main doorways of both of the two structures
built of fine Inka masonry. These alignments are associated to positions of the Sun in
the equinox, zenith, antizenith and solstices; alignments of the Moon in their extre-
me positions; as well as very specific star positions of Alpha and Beta Centaurus and
the Southern Cross. With these evidences, we propose the importance of the use of
the Inka Astronomy in the provincial centers.

Keywords Tawantinsuyu, Inka astronomy, ushnu, space organization, regional calendars.

\footnotetext{
* Arqueólogo de la Universidad Nacional Mayor San Marcos, miembro fundador del Centro de Investigación de Arqueología y Antropología Andina "Wamani". Correos electrónicos: josepinomatos@hotmail.com, arqueologia@peru.com
} 
"Estos observatorios determinaban el comienzo de las labores agrícolas, especialmente el barbecho, el riego y la siembra. Se creía que si se dejaba pasar el momento oportuno, la cosecha de maíz peligraba". John V. Murra 1978:42.

\section{Introducción}

La astronomía tuvo un rol fundamental en el desarrollo de las sociedades andinas prehispánicas, sobre todo en las actividades económicas y religiosas. Esta importancia se manifiesta de manera especial en la agricultura y los rituales asociados a ella. Para el caso de la sociedad Inka, se cuenta con un importante corpus de información etnohistórica y arqueológica (Aveni 1993; Bauer y Dearborn 1998; Hyslop 1985,1990; Urton 1981,1985; Ziolkowski y Sadowski 1992; Zuidema 1977). Los estudios sobre astronomía Inka se han realizado, en su mayor parte, en el Cuzco, ciudad que fue la capital del imperio del Tawantinsuyu, donde existieron varias estructuras con funciones astronómicas que los españoles destruyeron en el proceso de evangelización (Aveni 2001:312; Bauer y Dearborn 1998:124). Estas estructuras fueron principalmente: el Ushnu, las Sukankas, el Sunturwasi, el Corikancha (Aveni 2001:312-313; Zuidema 1981:319-324); además muchas de las wakas del sistema radial de ceques (Zuidema 1977); de las cuales sólo existen en la actualidad el Corikancha y algunas de las wakas del sistema de ceques. Los fenómenos astronómicos que observaron los Inkas en el Cuzco, siguiendo de forma sistemática el movimiento anual del Sol por el horizonte fueron los solsticios, equinoccios, pasos del Sol por el zenit y antizenit (nadir), la Luna en sus diferentes fases y sus posiciones extremas norte y sur, las estrellas (principalmente Alfa y Beta Centaurus), las constelaciones de las Pléyades y la Cruz del Sur (Zuidema 1981,1982; Urton 1985; Zuidema y Urton 1976). En el caso del Cuzco son las fuentes etnohistóricas las que brindan la mayor parte de la información.

Tom Zuidema conjuntamente con Anthony Aveni, realizaron estudios sobre astronomía Inka durante varias temporadas en Cuzco y alrededores. Estos investigadores presentan ciertas propuestas sobre los lugares de observación, ubicación de los marcadores de horizonte y las direcciones de los alineamientos, los cuales estarían asociados a solsticios, pasos del Sol y la Luna por el zenit y antizenit; como también a la constelación de las Pléyades (Zuidema 1981,1983; Aveni 2001). Entre estas propuestas destacaría la hipótesis sobre la importancia que tuvo en la sociedad Inka el registro de las fechas del paso del Sol por el zenit y antizenit (Zuidema 1981:322-324), propuesta que generaría debate al no existir evidencias físicas (de observatorios y marcadores de horizonte) relacionados a dichos fenómenos (Bauer y Dearborn 1998:120-121). Sin embargo, la mayoría de crónicas describen muchos aspectos de la astronomía Inka, e incluso algunas de ellas hacen referencia con más detalle, sobre algunas construcciones que posiblemente sirvieron para estas funciones, como también, de personajes encargados de estas observaciones.

La crónica de Guaman Poma presenta información sobre un astrólogo indígena llamado Juan Yunpa, que conocía las posiciones del Sol en el horizonte a lolargo del año, al observar sus ortos y ocasos. Éste hacía uso de marcadores solares denominados cillas (asientos) donde el Sol se ubicaba de acuerdo a las fechas previstas, teniendo cada mes o periodo su cilla correspondiente; enfatizando la posición del Sol en la cilla correspondiente a agosto, como referente para observar el movimiento de este astro. Asimismo, este cronista da a entender el uso de ventanas, luz y sombras en construcciones para efectuar observaciones astronómicas, con lo cual podían tener fechas más precisas que indicaban el tiempo para realizar actividades agrícolas y ganaderas (Guaman Poma 1980:884[898]). Guaman Poma, también presenta un dibujo del referido astrólogo, que aparece sosteniendo en su mano izquierda 


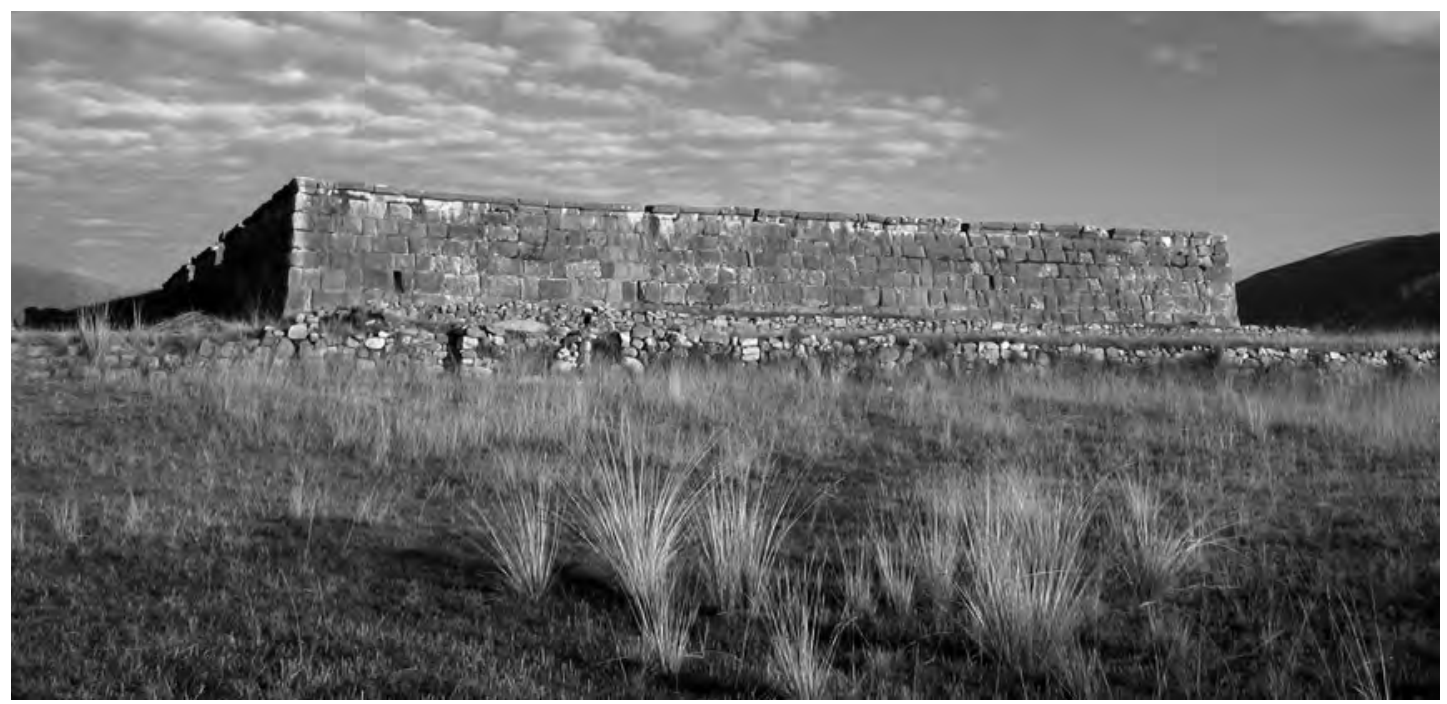

El Ushnu de Huánuco Pampa.

un khipu, que posiblemente serviría para llevar cuentas a manera de calendario (Guaman Poma 1980:883 [897]), función que tenía también un secretario oficial del Inka llamado quilla uata quipoc, encargado de llevar la cuenta de los meses y años; quien también esta descrito gráficamente con un khipu (Guaman Poma 1980: 358[360],359[361]).

El Tratado de Francisco de Ávila, sobre Huarochirí, hace referencia a unos personajes conocidos como Yañca, que determinaban la fecha en que se debía ir a adorar al dios Pariacaca, utilizando construcciones para observar el Sol, haciendo uso de efectos de luz y sombra en las observaciones astronómicas: “...estos hombres observan el paso del Sol desde un muro construido según reglas muy precisas [esto es la sombra que va haziendo la pared con el sol]. Cuando el Sol alcanza el muro en cuestión, dicen a la gente que ha llegado el día o que había que esperar al día siguiente" (Taylor 1999:125). Ávila, refiriéndose a la fiesta que realizaban en abril en honor de Paricaca, describe que estos yañac (o Yañca), se "sientan" en un determinado lugar y observan la salida del Sol en "...cierta parte de un cerro que ya tienen marcada" (Taylor 2000:82), sólo así sabían con precisión el día que tenían que realizar la fiesta a esta deidad.
De la misma forma, Juan de Betanzos, menciona un sistema de registro de fenómenos astronómicos conocido como Pacha Unan Chac, que servía para que no perdiesen la cuenta de los meses y las fechas en las que tenían que sembrar y hacer las fiestas; y que funcionaba mediante la observación del Sol en las mañanas y tardes, todos los meses del año, para saber las temporadas de siembra y cosecha; este sistema constaba de ciertas construcciones ubicadas en las cimas de los cerros por donde se miraba salir y ponerse el Sol (Betanzos 1987:70-74).

Existen muy pocos estudios sobre astronomía Inka para las áreas provinciales del Tawatinsuyu, sin embargo la evidencia arqueológica presenta un mayor potencial, sobre todo en los tampus con carácter de centro administrativo en las capitales de los wamanis o provincias (Hyslop 1985,1990; Matos 1986,1994; Ziolkowski y Sadowski 1992). Uno de estos tampus fue "Huánuco Pampa" ubicado en la sierra central del Chinchaysuyu, a una altitud de 3,700 msnm, y que posee una relación con los equinoccios por tener un eje este-oeste que cruza el sitio e influye en la organización espacial del Tampu (Harth Terre 1964; Morris 1987; Morris y Thompson 1970,1985). 
Nuestra reciente investigación realizada en "Huánuco Pampa" muestra nuevas evidencias de alineamientos y presenta propuestas sobre el tipo de observaciones astronómicas que realizaron los Inkas en ese tampu, los principios de organización espacial basados en orientaciones astronómicas, así como la utilización especifica de algunas estructuras como observatorios. Las estructuras presentadas como observatorios son: la plataforma ushnu y una estructura conocida en el lugar como el inkawasi. El ushnu, conocido en el imperio como lugar de libaciones y observaciones astronómicas (Zuidema 1989:402-454; Hyslop 1990:69-101, Pino 2001a), presenta además en Huánuco Pampa, características de un observatorio astronómico complejo, que funcionaba mediante un sistema de asientos y accesos. El inkawasi (estructura de composición dual y cuatripartita), presenta en este caso, un diseño especial para la observación de fenómenos astronómicos, que funciona por efectos de luz y sombra entre sus componentes internos (como accesos y nichos), utilizados para una mayor precisión en la determinación de ciertas fechas. La información arqueológica aquí presentada, respalda ciertas propuestas sobre la astronomía Inka, difíciles de comprobar en el Cuzco debido a la destrucción de la evidencia material.

\section{Metodología de la investigación}

El objetivo principal de la investigación fue el detectar alineamientos (conformados por estructuras, elementos de la arquitectura, y formaciones naturales del paisaje local), asociados a fenómenos astronómicos significativos o de especial importancia para la sociedad Inka. Se utilizó para estos fines la información, planos y sectorización publicada por Morris y Thompson (1970, 1985) y Shea (1966), luego se elaboraron planos de mayor precisión de dos estructuras: el ushnu en el sector central y el inkawasi en el

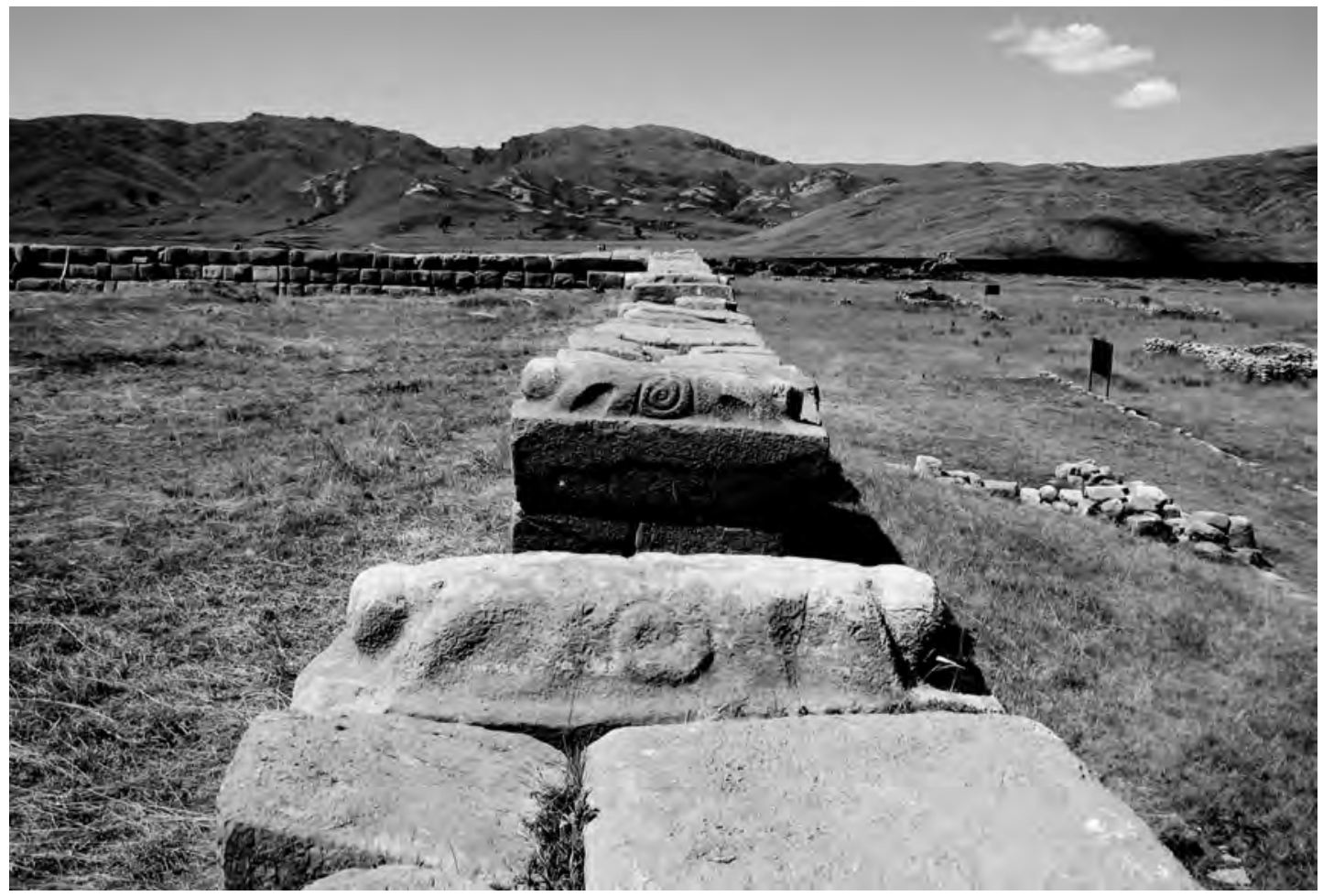

Felinos tallados en los accesos del ushnu. 
sector oriental. Asimismo, para la determinación del norte verdadero y el registro de los alineamientos, usamos un teodolito Kern DKM2-A con precisión de un segundo de arco. El norte verdadero se obtuvo mediante observaciones solares, y se corrigió en base a las posiciones verdaderas del Sol obtenidas con el programa HORIZONS Ephemerides Generator de la NASA y el Jet Propulsion Laboratory. Las correcciones de refracción atmosférica y paralaje fueron hechas utilizando las formulas y constantes publicadas por Meeus (2000a:105,279).

Para nuestro estudio, utilizamos el termino "alineamiento astronómico" como la línea orientada hacia un fenómeno astronómico (para este caso, posiciones de orto, ocaso y zenit, de los astros), desde y hacia referentes físicos tangibles (llámese punto de observación y marcadores de horizonte), estos alineamientos pueden estar compuestos por estructuras, conjuntos de estructuras, accesos principales, calles, caminos, y horizontes naturales del paisaje local, especialmente cimas y laderas de cerros. Y definimos "observatorios", como las estructuras que guardan en su diseño interno características especia les para la observación de fenómenos astronómicos. Estas construcciones pueden presentar uno o varios alineamientos astronómicos, formados por sus elementos internos (accesos, ventanas, nichos, corredores, etc.).

Los fenómenos astronómicos asociados a los alineamientos registrados en "Huánuco Pampa", son los que se nombran en la tabla 1 , simbolizados con las siglas entre paréntesis.

\section{Evidencias}

Los Alineamientos Astronómicos serían de dos tipos:

- a nivel de asentamiento, $y$

- a nivel de estructuras.

\section{a) Alineamientos a nivel de asentamiento}

Partían del centro del sitio, específicamente de la plataforma conocida como ushnu, situada en medio de la plaza. Estos alineamientos estarían asociados a solsticios, equinoccios y posiciones extremas de la Luna, y están orientados a elementos específicos de la arquitectura, especialmente a accesos y esquinas de los conjuntos de estructuras, comotambién a calles principales (Figura 1).

Tabla 1. Fenómenos astronómicos asociados a alineamientos en Huánuco Pampa.

1. Salida del Sol en el Solsticio de Diciembre.

2. Puesta del Sol en el Solsticio de Diciembre.

3. Salida del Sol en el Solsticio de Junio.

4. Puesta del Sol en el Solsticio de Junio.

5. Salida del Sol en el Equinoccio.

6. Puesta del Sol en el Equinoccio.

7. Salida del Sol el día de su Paso por el Zenit.

8. Puesta del Sol el día de su Paso por el Zenit.

9. Salida del Sol el día de su Paso por el Anti-Zenit.

10. Puesta del Sol el día de su Paso por el Anti-Zenit.

11. Salida de Alfa, Beta Centaurus y la Cruz del Sur.

12. Puesta de Alfa, Beta Centaurus y la Cruz del Sur

13. Salida de la Luna en su Posición Extrema Norte.

14. Puesta de la Luna en su Posición Extrema Norte.

15. Salida de la Luna en su Posición Extrema Sur.

16. Puesta de la Luna en su Posición Extrema Sur.
( SSSD )

(PSSD)

( SSSJ)

(PSSJ)

(SSEQ)

(PSEQ)

(SSPZ)

(PSPZ)

(SSPAZ)

(PSPAZ)

(S-ABC,CS)

(P-ABC,CS)

(SLPEN)

(PLPEN)

(SLPES)

(PLPES) 


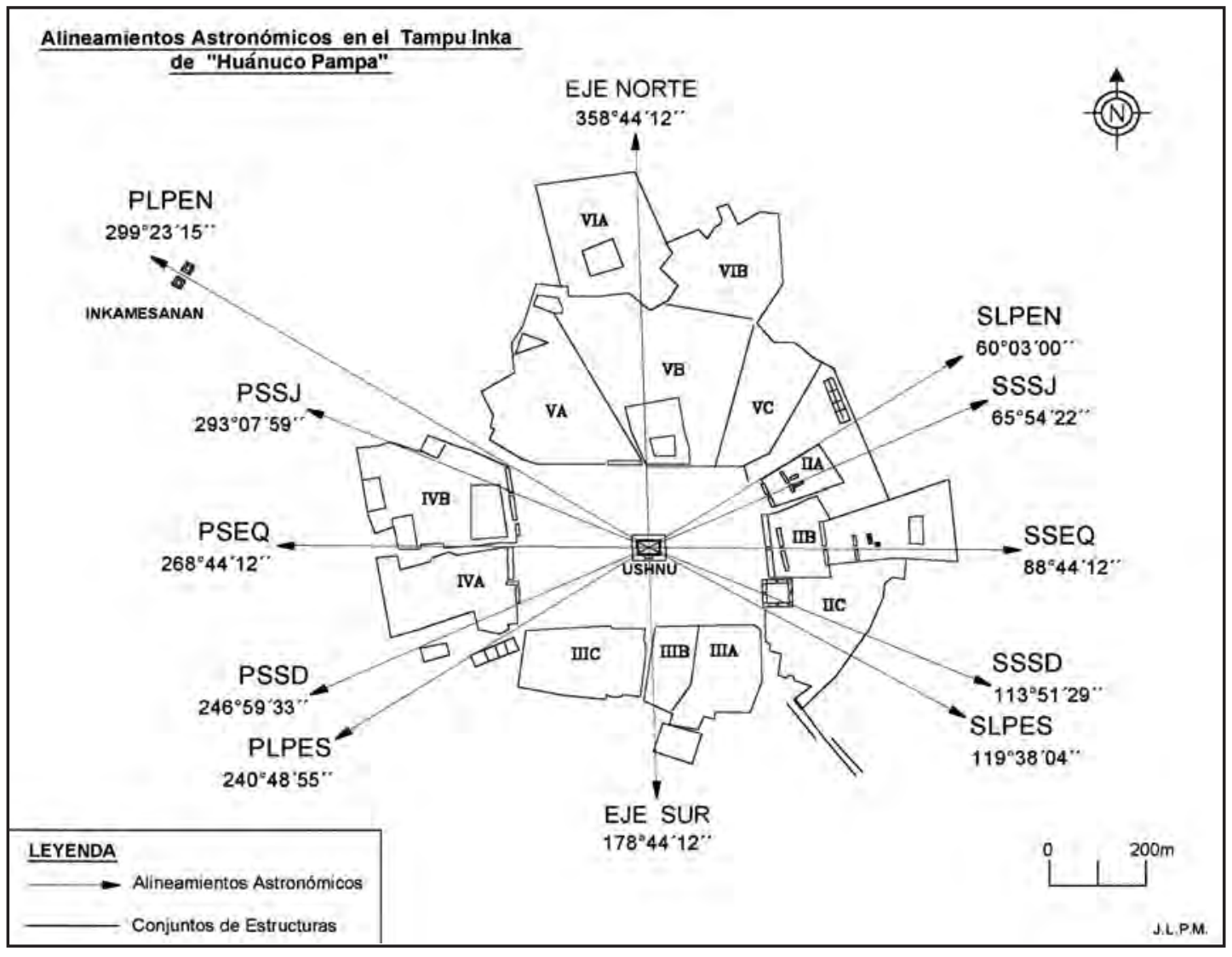

Figura 1. Alineamientos a nivel de asentamiento.

Los alineamientos hacia el lado Este se dirigen hacia los siguientes fenómenos astronómicos:

- SLPEN, por el lado Norte del sector IIA.

- SSSJ, a través del acceso principal del sector IIA.

- SSEQ, a través de las cuatro portadas que cruzan el sector IIB, en dirección a un lugar llamado Inkanan, ubicado en los cerros que sirven de horizonte al Este.

- SSSD, a través del acceso principal de la kancha ortogonal del sector IIC.

- SLPES, por la esquina Sur-Oeste de la kancha ortogonal del sector IIC.

Y hacia el lado Oeste dirigidos a:

- PLPEN, a través de la calle Nor-Oeste, y entre las dos plataformas conocidas como Inkamesanan, ubicadas a la salida del tampu, en el camino hacia el
Norte con dirección al Tampu de Taparaku. - PSSJ, a través del acceso principal hacia el sector IVB.

- PSEQ, a través de la calle Este.

- PSSD, por el lado Sur de la estructura Sur del sector IVA ubicada frente a la plaza.

- PLPES, a través de la calle Sur-Oeste.

Existen además, orientaciones hacia:

- El Sur, que se dirige hacia la calle Sur y

- El Norte, que se dirige hacia la esquina SurOestedelAcllawasi.

Estas ultimas orientaciones posiblemente, las determinaron tomando la mitad de arco (la bisectriz) del ángulo formado por los alineamientos de S-ABC,CS y P-ABC,CS, que trazaran desde el ushnu, debido a que estos alineamientos estelares determinan una mayor precisión y son de observación másfactible. 


\section{b) Alineamientos a nivel de estructuras}

Como señalamos antes, estas se manifiestan principalmente en dos estructuras:

-El Ushnu (Lat. 0952'39"S, Long. 076 49' 00" W; $3666 \mathrm{msnm})$.

Situado en el sector central del tampu, es una gran plataforma rectangular (de 32 x $48 \mathrm{~m}$ de planta y $4 \mathrm{~m}$ de altura aproximadamente), construida en piedra labrada al estilo cuzqueño, a la cual se accede por su lado sur a través de una escalinata y dos accesos (con medidas promedio de $3 \mathrm{~m}$ de largo y $1.40 \mathrm{~m}$ de ancho). La parte superior está compuesta por muros a manera de parapetos (de $1.30 \mathrm{~m}$ de altura) y diez espacios, a manera de nichos, que miran hacia el interior de la plataforma, y son conocidos como tianas por usarse como asientos. Estas tianas (de forma rectangular, con medidas de $2 \mathrm{~m}$ de largo, $0.80 \mathrm{~m}$ de altura y $0.50 \mathrm{~m}$ de profundidad) han sido edificados en el lado interior de los muros Norte, Este y Oeste (Figura 2).

Las tianas o asientos de piedra y los accesos se utilizaron para la conformación de los alineamientos. Estos se formarían al orientarse la vista de una tiana hacia la otras (algunas opuestas perpendicularmente y otras de manera diagonal). La mayoría atravesaría la parte central de la estructura ( Figura 3). Estos alineamientos se dirigen hacia los fenómenos de SSSD, PSSD, SSSJ, PSSJ, SSEQ, PSEQ, SSPZ, PSPZ, SSPAZ, PSPAZ, S-ABC,CS, P-ABC,CS, SLPEN, PLPEN, SLPES y PLPES (Cuadro 1). Esto sugiere convincentemente que las personas encargadas de las observaciones, pudieron realizarlas situandose en las tianas y orientandose hacia las paredes opuestas, con dirección a determinadas tianas opuestas y los accesos al ushnu. Aquí era posible ubicar puntos específicos en el horizonte natural del paisaje que servirían en la observación de los fenómenos astronómicos.

- El Inkawasi (Lat. 0952'31"S, Long. 076 48'43"W; 3644 msnm), ubicado en el Sector IIB, es una estructura doble, de planta rectangular (de $9 \times 23 \mathrm{~m}$ ), compuesta por dos partes iguales (norte y sur) divididas por un corredor central que lo atraviesa en un sentido SW-NE. Cada parte de la estructura está dividida a su vez en dos recintos por un muro medianero (Figura 4) y cuenta con accesos de forma trapezoidal que

Cuadro1: Alineamientos astronómicos registrados en el "Ushnu".

\begin{tabular}{|c|c|c|c|c|}
\hline \multicolumn{2}{|c|}{ ^ääåÉ ããÉE̊åíç } & \multirow{2}{*}{ ^òáãìí } & \multirow{2}{*}{$\begin{array}{l}\text { bäÉ̂̂ Åá } 1 / 4 a ̊ \\
\text { ecêáòcåí́E }\end{array}$} & \multirow{2}{*}{$\begin{array}{l}\text { cÉå1/4ãÉåç } \\
\text { ^ëç̊̊á Çç }\end{array}$} \\
\hline aÉW & ${ }^{\wedge} \mathrm{W}$ & & & \\
\hline $\mathrm{qkO}$ & $\mathrm{qbN}$ & NQœOT"RM"'” & MOœOR"OM"'" & pppa \\
\hline $\mathrm{qkP}$ & $\mathrm{qtN}$ & OQRœQV"OM"'" & MRœNU"RO"', & mppa \\
\hline $\mathrm{qtN}$ & $\mathrm{qkP}$ & SRœQV"OM"'” & MPœMT"OM'"' & pppg \\
\hline $\mathrm{qbN}$ & $\mathrm{qkO}$ & OVQœOT"RV'”' & MPœMQ"MV"”' & mppg \\
\hline $\mathrm{qtO}$ & $\mathrm{qbO}$ & UUœQQ"NO"”' & MOœQR"OR"'” & ppbn \\
\hline $\mathrm{qbO}$ & $\mathrm{qtO}$ & OSUœQQ"NO"”' & MQœMT"RP"'” & mpnb \\
\hline $\mathrm{qtN}$ & $\mathrm{qbP}$ & VVœPR"NV'”' & MOœNT"NM"'" & ppmw \\
\hline $\mathrm{qbN}$ & qtP & OSMœMS"RM""' & MOœQU"NT"'" & mpmw \\
\hline qtP & $\mathrm{qbN}$ & UMœMS"RM"'” & MOœRP"QR"” & $\mathrm{ppm}_{\mathrm{w}}$ \\
\hline $\mathrm{qbP}$ & $\mathrm{qtN}$ & OTVœPR"OM"”' & MQœRN"PT"”" & mpnow \\
\hline $\mathrm{qkN}$ & ${ }^{\wedge} \mathrm{pO}$ & NRQœMM"PQ"'” & MQœMO"RM"'" & $\mathrm{pJ}^{\wedge} \mathrm{I}^{\wedge} \mathrm{p}$ \\
\hline qkQ & ${ }^{\wedge} \mathrm{pN}$ & OMPœOO"MM"', & MUœOO"MO"”' & $\mathrm{mJ}^{\wedge}{ }^{-} \mathrm{I} p$ \\
\hline $\mathrm{qtO}$ & $\mathrm{qkQ}$ & SMœQM"OV"”' & MPœNU"OM"'" & pimbk \\
\hline $\mathrm{qbN}$ & $\mathrm{qkP}$ & OVVœMV"NM"”' & MPœMR"NM"'” & mimbk \\
\hline $\mathrm{qkP}$ & $\mathrm{qbN}$ & NVœMV"NM"'" & MOœOQ"RQ"'” & pimbp \\
\hline qkQ & $\mathrm{qtO}$ & OQMœ=QM"OV"", & MTœQS"MQ"'" & $\operatorname{mimbp}$ \\
\hline
\end{tabular}






Figura 2. Plano de la parte superior de la plataforma Ushnu.



Figura 3. Orientaciones astronómicas en el Ushnu. 
Figura 4. Plano de la estructura Inkawasi.

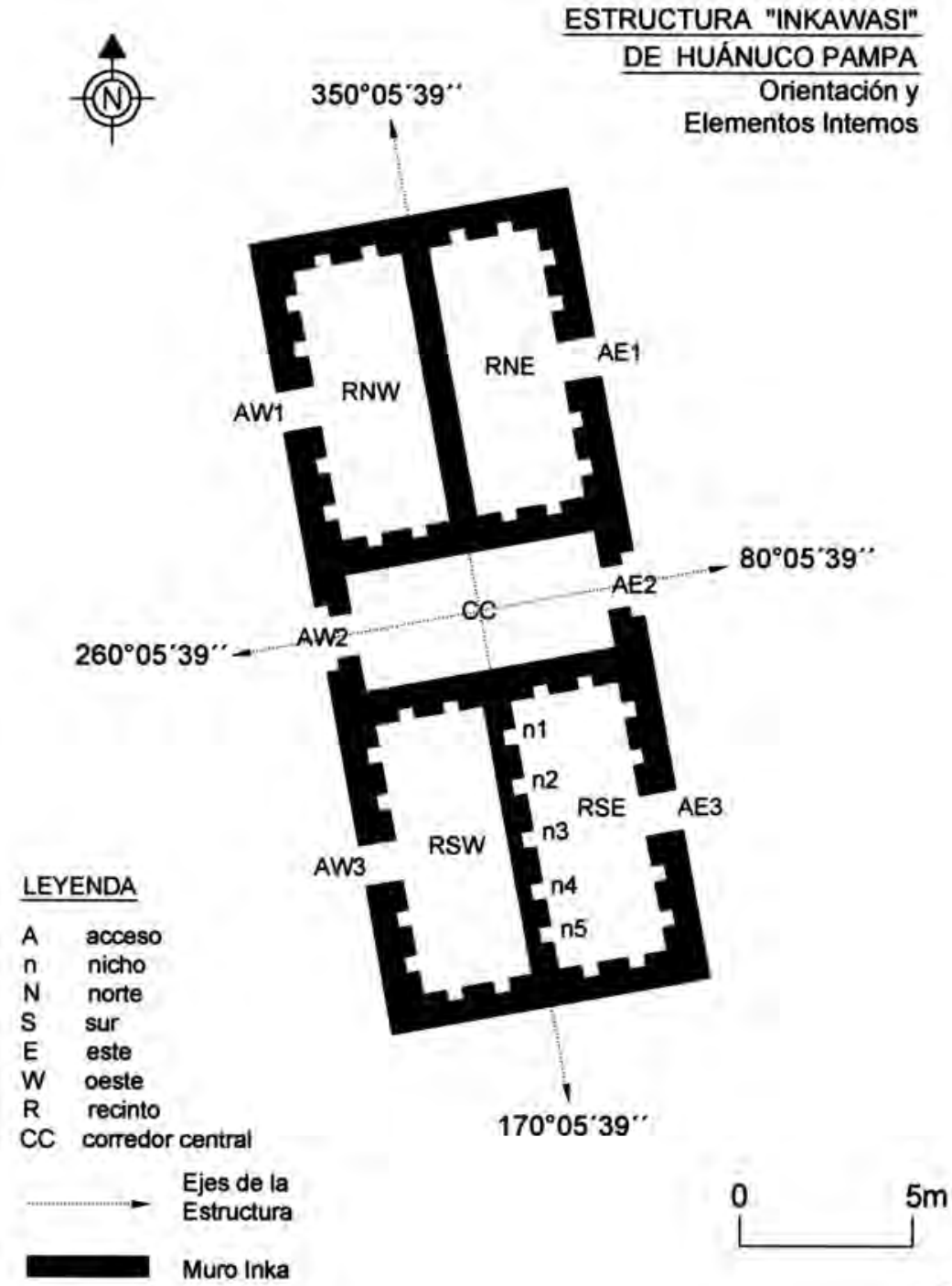

J.LP.M están hacia los lados Este y Oeste (con medidas promedio de $1.20 \mathrm{~m}$ de base, $2.35 \mathrm{~m}$ de altura y $1.05 \mathrm{~m}$ de ancho en el umbral). En el interior de los muros de cada recinto se encuentran 13 nichos trapezoidales (cada nicho con un promedio de medidas de: $50 \mathrm{~cm}$ de base, por $50 \mathrm{~cm}$ de altura y $35 \mathrm{~cm}$ de profundidad), a una altura de $1.90 \mathrm{~m}$ desde el nivel del suelo. Los nichos y los accesos principales de los recintos sirven para la conformación de los alineamientos, dirigiéndose de partes especificas de los nichos hacia el centro y partes especificas de los accesos. En el corredor central se utilizaron los centros de los accesos y las esquinas exteriores de los mismos (Figura 5). Los alineamientos se dirigen hacia los fenómenos de SSPAZ, SSPZ, PSPZ, SSEQ, PSEQ, SSSJ, SSSD, SLPEN y SLPES (ver cuadro 2). La evidencia sobre alineamientos en los recintos se basa en el registro llevado a cabo en el Recinto Sur-Este, por estar en mejores condicio- 


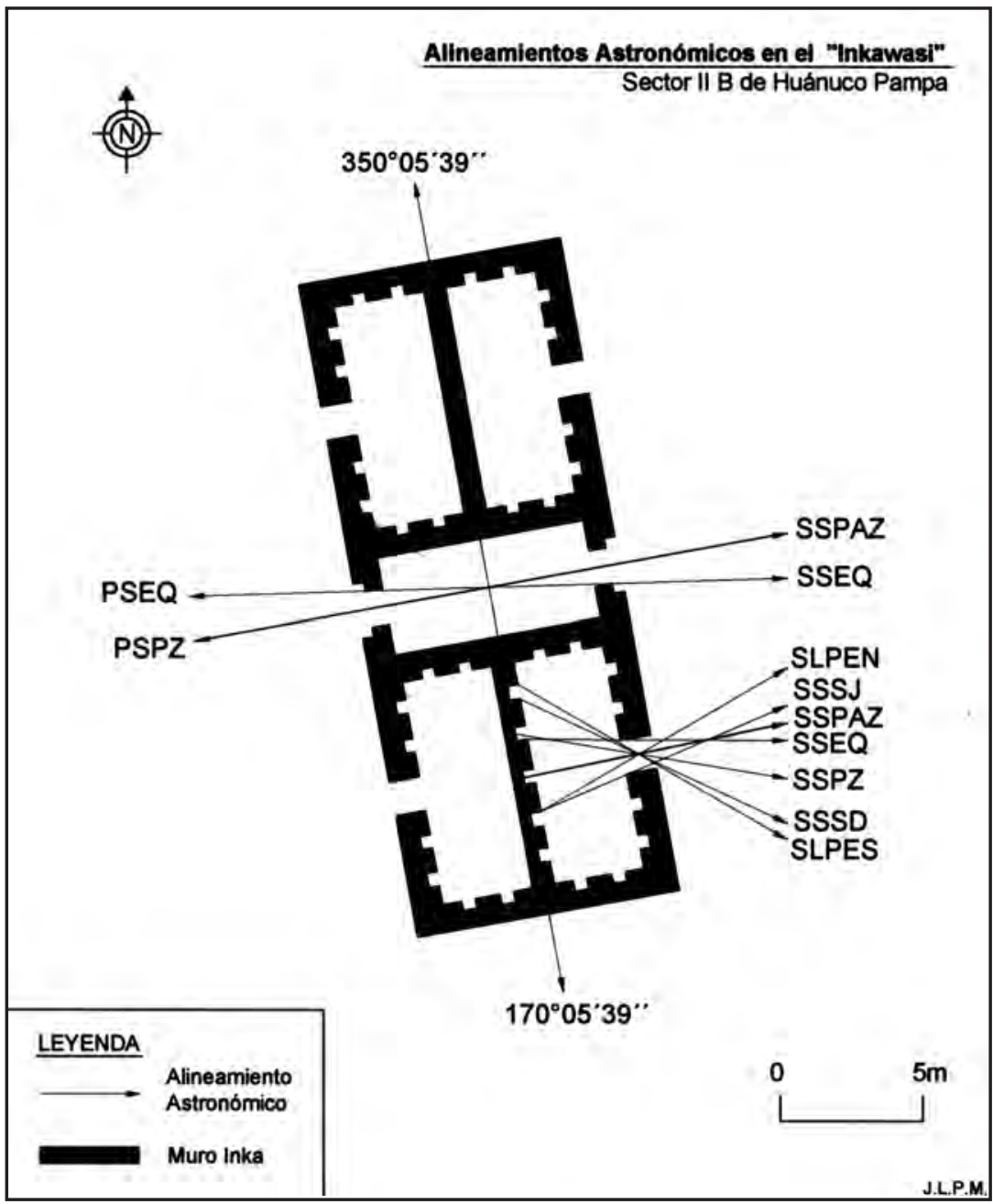

Figura 5.

Orientaciones astronómicas en el Inkawasi.

Cuadro 2: Alineamientos astronómicos registrados en el Inkawasi.

\begin{tabular}{|c|c|c|c|c|}
\hline \multicolumn{2}{|c|}{ ^äáåÉ ãáÉåíç } & \multirow{2}{*}{ ^ òáã ì í } & \multirow{2}{*}{$\begin{array}{l}\text { bäÉ̂̂i Åá1/4å } \\
\text { eçêáòçåáíÉ }\end{array}$} & \multirow{2}{*}{ 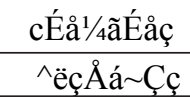 } \\
\hline aÉW & ${ }^{\wedge} \mathrm{W}$ & & & \\
\hline bëèìáå =ÉñíÉêáçê $=$ ëiê $=C \zeta E ́=$ & Éåîeç=ÇÉbP & NQœRQ"PP & "'MQœPV"OT", & pppa \\
\hline bëèíå $\sim=$ ÉñíÉêáçê=åçêíÉ=ÇÉ= & 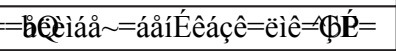 & SSœRP"'MP', & "MPœRP"NM", & pppg \\
\hline bëèiàå $=$ ÉñíÉêáçê $=$ ëiê $=C \zeta E ́=$ & (Đëèiáå =áåíÉêáçê=åçêíí $₫ \emptyset ̧ \mathbb{E}=$ & UUœQP”MR & '"MOœRU"OMI', & " ppbn \\
\hline Éåíêç̧=áåíÉếçêe=ÇÉ=åO & Éåîêç=ÇÉbP & VVœNM"Q & "MOœON"PP" & ppmw \\
\hline Éåíêç=áåí́êéççê=ÇÉ=åP & Éåîę̧=ÇÉbP & UMœMR"P & "MOœQV"QR" & $" \quad \mathrm{ppm}^{\wedge} \mathrm{w}$ \\
\hline bëèiáå =ÉñníEéáçê=åçêíí=ÇÉ= & Éåîêç=ÇÉbP & SMœNT"RS' & "'MPœQM"OS", & $"$ pimbk \\
\hline bëèiáå =Éñííêấçê=åçêî́É=ÇÉ= & Éåîêç̧=ÇÉbP & NVœRO"PN & "'MRœNU"Mф' & " pimbp \\
\hline 'Éåîeç=ÇÉ=O & Éåíęc=ÇÉbO & UMœMR"P & "'MOœQM"RR' & $" \mathrm{ppm}^{\wedge} \mathrm{w}$ \\
\hline Éåíêç=ÇÉbO & Éåîeç=ÇÉचO & OSMœMR"P & V"'JJJ=JJJ=JJJ & mpmw \\
\hline 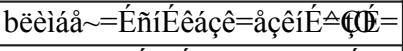 & bëèiàå =Éñí́éáçê=ëîê=டுல́= & UUœQP”OM & ["MOœQT"RN"' & ppbn \\
\hline 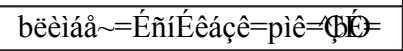 &  & OSUœQP"OA & Q'”'JJJ=JJJ=JJJ & mpbn \\
\hline
\end{tabular}


conservación. Para llevar a cabo las observaciones astronómicas, las personas encargadas tuvieron que situarse al pie de los nichos o de partes especificas de estos, como esquinas y centros, orientándose siempre hacia los accesos, especialmente hacia la parte central y esquinas exteriores de los mismos. Estos alineamientos tendrían como objetivo ubicar puntos específicos en el horizonte natural de los cerros demarcados por los accesos mencionados. A su vez, en esta estructura se utilizaron el efecto de luz y sombras para una observación más precisa en algunos casos, donde las esquinas exteriores de los accesos proyectaban líneas de sombra que coincidían con las esquinas exteriores de los nichos al momento de entrar la luz del Sol en las salidas o puestas de los mismos, con lo cual las observaciones podían realizarse sólo viendo este efecto desde cualquier parte del interior del recinto usado, sin la necesidad que el observador esté orientado hacia el astro durante el fenómeno astronómico.

El registro de ángulos de azimut y elevación en ambas estructuras es presentado en los Cuadros 1 y 2 , en base a los cuales se percibe cierto grado de precisión y sistematización al momento que debieron construir las estructuras y definir sus funciones astronómicas.

Utilizando los principales alineamientos solares en Huánuco Pampa, los Inkas contaron con los marcadores necesarios para controlar los 365 días del año (Figura 6). Las fechas calculadas para los alineamientos solares presentes en Huánuco Pampa, para el año 1530 según el Calendario Gregoriano, serían:

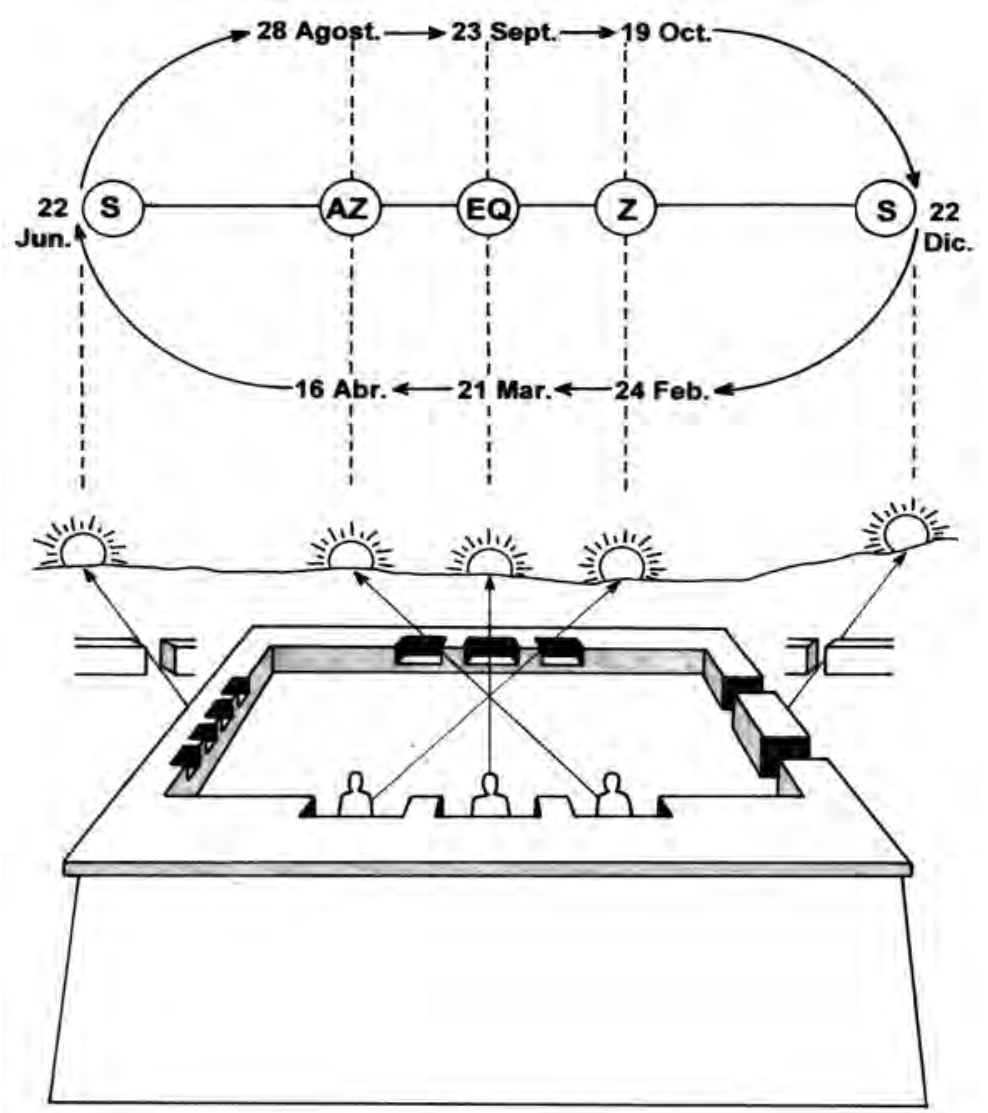

Figura 6. Sistema de Control del año Solar utilizando el Ushnu de Huánuco Pampa.

-Solsticio ( S ): 22 de diciembre. -Zenit (Z): 24 de febrero. -Equinoccio (EQ): 21 de marzo. -Anti-Zenit (AZ): 16 de abril.

-Solsticio (S): 22 de junio.

-Anti-Zenith (AZ): 28 de agosto. -Equinoccio (EQ): 23 de setiembre. -Zenit (Z): 19 de octubre.

Las fechas del año (según el calendario Gregoriano) en que ocurrieron los solsticios y equinoccios en la época Inka son casi las mismas que las de nuestra época, tal como lo demuestra un análisis sobre variaciones de tiempo de solsticios y equinoccios (desde el siglo XVI al año 1998), realizado por Meeus (2000b:346-352).

Otro aspecto es la ornamentación con relación a los fenómenos astronómicos. Se observa 
que en las principales áreas donde se evidencian los alineamientos astronómicos están presentes figuras de felinos tallados en pares. Específicamente a ambos lados de los accesos consecutivos que forman un eje equinoccial en el sector II-B; y a ambos lados de los dos accesos del ushnu relacionados a fenómenos estelares hacia el Sur.

\section{Propuestas}

\section{Marcadores astronómicos y diseño del Tampu}

Según nuestro registro, a nivel de asentamiento, creemos que para el trazado del Tampu, se ubicó primero un punto central donde se construiría el ushnu. Este punto serviría para determinar los principales alineamientos astronómicos y serviría como eje para el diseño de todo el asentamiento. En este diseño funcionarían como marcadores astronómicos los accesos y calles principales que dan a la plaza, así como rasgos de la arquitectura de algunos conjuntos de estructuras. Diseños similares son observados en otros sitios Inka, como Pumpu en Junín (Matos 1986), Inkawasi en Cañete (Hyslop 1985), y otros tampus de la sierra central (Pino 2003).

Cabe resaltar el lugar conocido como Inkamesanan al Nor-Oeste del asentamiento, donde se encuentran dos pequeñas plataformas dispuestas a cada lado del camino Inka que va hacia el norte. Entre estas dos plataformas pasa un alineamiento relacionado con la Luna en su posición extremo Norte. Estas plataformas sirvieron posiblemente como un referente astronómico observable desde el ushnu. En Tikani, Isla del Sol, en Bolivia, se encuentran dos estructuras similares que servían de referentes astronómicos y eran observadas también desde una plataforma, pero relacionadas a la puesta del Sol en el solsticio de junio (Dearborn etal. 1998:250-252).

\section{Observatorios}

Proponemos que el ushnu y el inkawasi, funcionaron como observatorios astronómicos. El diseño complejo del ushnu posiblemente fue usado para observaciones astronómicas directas, pues esta estructura permite usar de marcadores astronómicos las tianas y los accesos. Las tianas cumplieron una doble función. Por un lado sirvieron para situarse en ellas como observador y, por otro lado sirvieron como marcador de referencia astronómica. Sirvieron también, en el caso del Sol, como asientos simbólicos para este astro, tal como es descrito en la crónica de Guaman Poma (1980: 884 [898]), con relación a las "cillas" del Sol

En cambio el diseño y orientación del inkawasi, sirvió para observaciones mediante efectos de luz y sombra, utilizando los accesos y los nichos. Esta forma de observación se encuentra descrita en los documentos etnohistoricos (Guaman Poma 1980:884[898]; Taylor 1999:125), y ha sido también identificada en estructuras estudiadas en Ingapirca, Ecuador (Ziolkowski y Sadowski 1992:48, 56-57), y Machu Picchu, Cuzco (Dearborn et al. 1987:347-350).

\section{Fenómenos astronómicos como referentes de} tiempo y espacio

Los alineamientos relacionados al Sol, Luna y estrellas, registrados en este tampu, permiten observar la forma de organización del "espacio" basándose en el "tiempo" y viceversa, usada por la sociedad Inka. Los fenómenos astronómicos de importancia, especialmente los más perceptibles a simple vista, sirvieron para organizar el calendario de actividades, a la vez que influyó en la organización del espacio, orientando físicamente la arquitectura hacia los alineamientos asociados con estos fenómenos (Pino 2001b).

Ese tipo de organización, responde al pensamiento tradicional andino de concebir tiempo y espacio como una unidad, y usar un solo término para describirlo: pacha, que define conceptos poli semánticos (Wachtel 1973:181). En el caso de Huánuco Pampa, fenómenos relacionados al Sol y la Luna, fueron usados posiblemente con más énfasis en la organización del tiempo, ya que los alineamientos habrían indicado las fechas 


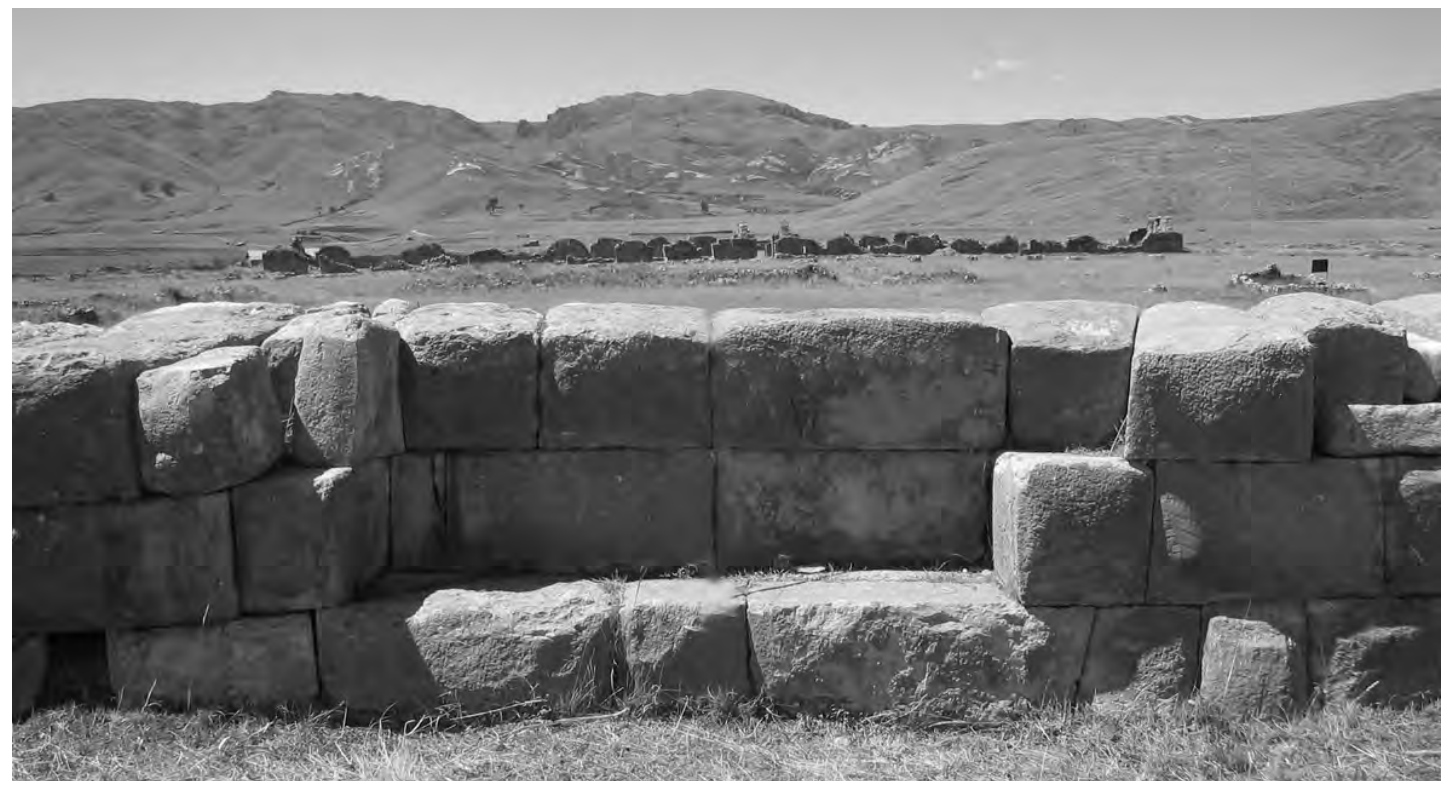

Tiana TE2 del Ushnu.

más importantes, mientras que las observaciones estelares habrían servido para ubicar con más precisión el eje Norte-Sur, y diseñar el asentamiento.

\section{Huánuco Pampa y la posible reconstrucción de calendarios provinciales}

Según las evidencias de alineamientos solares presentes en este tampu, proponemos dos "sistemas de control de los días del año":

a) El primero estuvo basado en las observaciones del paso del Sol por el zenit y anti-zenit. Especialmente usando las fechas del Sol en el zenit, fenómeno que sucede dos veces al año (19 de octubre y 24 de febrero, en Huánuco Pampa) y que es percibido estos días mediante la observación de un gnomon que no proyecta sombra al mediodía. Este fenómeno divide el año en dos partes, con periodos de notables diferencias en números de días ( 237 y 128 días). Si se utilizan los referentes del anti-zenit de agosto -asociado al inicio del calendario agrícola, principalmente de la siembra del maíz (Zuidema 1981) - y el zenit de febrero, podemos tener dos periodos casi homogéneos de 180 y 185 días. Además en el caso del maíz, la siembra tenía que ser muy precisa y sus xo marcadores astronómicos bien definidos. Así lo menciona Guaman Poma en la parte de su crónica referente a agosto: "Y ci hierra un mes una semana o un día del ruedo y rreloxo que lo ven los viejos, se daña el maýs. Quiere entrar en el punto conquauidad del sol y ayre del cielo la comida. Y ací quiere Dios que entre el maýs en su tiempo" (Guaman Poma 1980:1152 [1162]).

Este sistema, al fijar la posición en el horizonte del orto y ocaso del Sol en su paso por el zenit (que va de acuerdo a la latitud del sitio) y certificar que se trata de esos días mediante la observación de construcciones que sirvan como gnomones o viceversa sería muy seguro en el conteo de días. Las posiciones del Sol en el anti-zenit estarían fijadas por oposición a las del zenit (a 180 grados) tal como lo propuso Zuidema (1981:324). Es decir, al tomar la posición de salida del Sol en el zenit se invierte esta dirección y se obtiene la posición del ocaso del Sol en el anti-zenit y, de la misma manera, ubicando la posición de la puesta del Sol en el zenit, se invierte la dirección y se obtiene la posición de orto del Sol en el anti-zenit. Además alrededor de las fechas del anti-zenit del Sol, la Luna llena en las noches pasa cercana al zenit. 
Este sistema podría haber sido complementado con un control de días, tomando como referente los equinoccios, ya que las fechas de estos fenómenos son cercanas en número de días (ver Cuadro 3).

Las evidencias en Huánuco Pampa se prestan para este postulado pues, en el Ushnu, 4 tianas (TE1,TE3,TW1 y TW3) corresponden a fechas del anti-zenit y zenit del Sol y 2 tianas centrales (TE2 y TW2) corresponden a fechas de los equinoccios (Figura 2 y 3). Asimismo, en el Inkawasi el corredor central y los accesos están orientados hacia la posición del Sol en las fechas del antizenit y zenit (ver figuras 4 y 5 ). b) El segundo estuvo basado en la división del año en dos pares de mitades: un par de mitades divididos por los equinoccios y el otro dividido por los solsticios. Se lograba además un mayor control, a través de un doble conteo de días del año: uno por mitades solsticiales y otro por mitades equinocciales. Se tenían así cuatro mitades de año (de 182, 183,186 y 179 días) con diferentes referentes astronómicos, que sumaban un total de 730 días, correspondientes a un doble conteo de 365 días (ver Cuadro 4).

Estos dos sistemas, pudieron haber funcionado de forma paralela. Un sistema para organizar las actividades agrícolas (anti-zenit, zenit); y el

Cuadro 3: Sistema calendárico de doble conteo anual de acuerdo a los alineamientos astronómicos solares registrados en Huánuco Pampa, teniendo como referencia el anti-zenit y los equinoccios.

\begin{tabular}{|c|c|c|c|c|}
\hline \multirow{10}{*}{ A } & Fenómeno Astronómico & $\begin{array}{l}\text { Fechas calculadas para } \\
1530 * *\end{array}$ & $\begin{array}{l}\text { Intervalo de días } \\
\text { entre Fenómenos }\end{array}$ & $\begin{array}{l}\text { Sum. de días por } \\
\text { mitades de año }\end{array}$ \\
\hline & Paso del Sol por el Anti-Zenit & Agosto 28 & 26 & \multirow{5}{*}{180} \\
\hline & Equinoccio & Septiembre 23 & 26 & \\
\hline & Paso del Sol por el Zenit & Octubre 19 & $\frac{20}{64}$ & \\
\hline & Solsticio & Diciembre 22 & 04 & \\
\hline & Paso del Sol por el Zenit & Febrero 24 & 64 & \\
\hline & Equinoccio & Marzo 21 & $\frac{25}{26}$ & \multirow{4}{*}{185} \\
\hline & Paso del Sol Por el Anti-Zenit & Abril 16 & $\frac{26}{67}$ & \\
\hline & Solsticio & Junio 22 & 81 & \\
\hline & Paso del Sol por el Anti-Zenit & Agosto 28 & 67 & \\
\hline & & & & $180+185=365$ \\
\hline \multirow{9}{*}{ B } & Equinoccio & Septiembre 23 & 26 & \multirow{5}{*}{179} \\
\hline & Paso del Sol por el Zenit & Octubre 19 & 64 & \\
\hline & Solsticio & Diciembre 22 & 64 & \\
\hline & Paso del Sol por el Zenit & Febrero 24 & 04 & \\
\hline & Equinoccio & Marzo 21 & 25 & \\
\hline & Paso del Sol Por el Anti-Zenit & Abril 16 & 26 & \multirow{4}{*}{186} \\
\hline & Solsticio & Junio 22 & 67 & \\
\hline & Paso del Sol por el Anti-Zenit & Agosto 28 & 67 & \\
\hline & Equinoccio & Septiembre 23 & 26 & \\
\hline & & & & $179+186=365$ \\
\hline \multicolumn{4}{|c|}{ Sumatoria total de días por doble conteo anual } & 730 \\
\hline \multicolumn{5}{|c|}{$\begin{array}{l}\text { * A: Año, que se divide y contabiliza en días, tomando como referente ña fecha del anti-zenit del Sol. } \\
\text { B: Año, que se divide y contabiliza los días tomando como referente los equinoccios. } \\
\text { ** Fechas correspondientes al Calendario Gregoriano, según la posición de Huánuco Pampa. }\end{array}$} \\
\hline
\end{tabular}


otro sistema (de solsticios y equinoccios) para organizar el tiempo con respecto a las festividades anuales y otras actividades.

El número de días existentes en los intervalos entre fenómenos astronómicos posiblemente fue contabilizado y registrado en khipus, tal como lo propone Gary Urton para el Khipu UR6 de la región de Chachapoyas, en el que los días se representan por cuerdas que conforman cuatro mitades de año (de 179,183, 185, y 183 días), sumando un total de 730 días. Según Urton este khipu sería un calendario bianual (Urton
2001:134-144). Al respecto, según las evidencias astronómicas de Huánuco Pampa, creemos que el khipu UR6 sí es en efecto, un registro calendario, pero se trataría de un calendario anual de doble conteo por mitades de año, intercalando dos sistemas de conteo: 1) Entre solsticios y entre equinoccios, 2) Entre fechas del antizenit y zenit del Sol y entre solsticios (o entre equinoccios); tal como se observa en los números de días existentes en las mitades de año, marcadas por alineamientos Solares de Huánuco Pampa y las cifras representadas en el KhipuUR6.

Cuadro 4: Sistema calendárico de doble conteo anual de acuerdo con los alineamientos astronómicos solares registrados en Huánuco Pampa, teniendo como referencia los solsticios y equinoccios.






\section{Astronomía e ideología Inka en Huánuco Pampa}

Las deidades principales de la sociedad Inka fueron en su mayoría astros, por lo que casi todos los aspectos de su cosmovisión están relacionados a ellos y plasmados en sus asentamientos y objetos. La relación más importante de esta sociedad fue con el Sol, astro considerado ancestro. La verdadera imagen física de esta deidad, elaborada por los Inkas, era la de un niño, que siempre estaba acompañado por dos serpientes y dos felinos a los costados. Sobre esta imagen del Sol, Tom Zuidema realizó un análisis, encontrando referencias en varias crónicas sobre un par felinos acompañando siempre al Sol (Zuidema 1974/76: 199-210). Asociada a esta concepción estaba la waka de Susurpuquio, donde al Inka (algunas crónicas mencionan a Pachakuti y otras a Wiracocha), el Sol se le reveló en sueños mostrándole su verdadera imagen bajo forma humana.

Esa waka según Zuidema estaba relacionada con el Solsticio de junio (Zuidema 1974/76: 212 221). Según Guaman Poma de Ayala en el Corikancha la imagen del Sol y la ventana por donde este astro proyectaba su luz estaban acompañadas de dos felinos a cada lado (orientados hacia el Sol):

"...en el tenplo de Curi Cancha, que todas las paredes alto y bajo estana uarnecida de oro finísimo y en lo alto del techo estana colgado muchos cristales y a los dos lados dos leones apuntando el sol. Alumbraua de las ventanas la claridad de los dos partes, soplauan dos indios y se c... Entrauan el viento del soplo y salía un arco que ellos les llaman cuychi. Y alli en medio se ponía el Ynga, hincado de rodillas, puesta las manos, el rrostro al sol y a la imagen del sol y decía su oración" (Guaman Poma 1980:262[264]-263[265]).

Según estas referencias, creemos que en Huánuco Pampa se trató de representar esta imagen del Sol con los felinos a los costados, especialmente para la fecha del equinoccio cuando los primeros y últimos rayos del Sol atraviesan simultáneamente todas las portadas con felinos tallados, ubicadas en el Sector IIB; efecto visual que la sociedad Inka usaría de forma simbólica posiblemente para mostrar su nexo filial con el Sol, ante las poblaciones conquistadas.

\section{Conclusiones}

El registro de evidencias de alineamientos astronómicos en Huánuco Pampa nos permite notar que la astronomía influyó mucho en el diseño de los tampus de este tipo. Existieron ciertas estructuras con funciones astronómicas para determinar posiciones de los astros en el horizonte en determinadas fechas, con lo cual se podía constituir un calendario, controlar el tiempo y organizar actividades de diferente tipo. El "Ushnu" y la estructura "Inkawasi" de "Huánuco Pampa" funcionaron como observatorios astronómicos, a la par que se realizaron en ellas otras actividades. Evidencias similares fueron estudiadas en otras partes del Tawantinsuyu (Zuidema 1980; Ziolkowski y Sadowski 1992).

La evidencia material y arqueoastronómica presentada también confirma ciertas hipótesis propuestas para el Cuzco, relacionadas al Paso del Sol por el Zenit y el Antizenith (Zuidema 1981:322-324); fenómenos astronómicos importantes para la agricultura sobre todo en las fechas concernientes a las épocas de siembra y cosecha.

El Ushnu de "Huánuco Pampa", además de servir de centro de todas las orientaciones y divisiones espaciales del tampu, posee un diseño interno especial relacionado con la mayoría de observaciones astronómicas (solares, lunares y estelares). Esto lo hace único en el Tawantinsuyu y pone en evidencia el uso de la astronomía de manera muy desarrollada en el diseño de los edificios que representaban símbolos de poder en la política de expansión imperial.

\section{Agradecimientos}

A Ramiro Matos del National Museum of American Indian - Smithsonian Institution por su estimulo, observaciones, y asesoría científica 
en nuestra investigación. A Julián Idilio Santillana, mi profesor de Arqueología Inka en la UNMSM por sus comentarios y generoso apoyo. A Gary Urton por su estímulo para con mi investigación. A Tom Zuidema por sus apreciaciones y críticas. A Mariuz Ziolkowski por sus comentarios y acertadas observaciones. A Manuel Perales por sus comentarios, sugerencias, bocetos artísticos y apoyo en toda la investigación. A Hernán Ramos por su valioso apoyo en el trabajo de campo, como también, por sus observaciones y correcciones al manuscrito original. A Dawn Denoncourt de la University of Florida, Martha Bell y Adam Stack de Yale University, por su invalorable ayuda en el trabajo de campo y el registro de alineamientos. A Eberth Serrudo por su gentil apoyo en las primeras etapas de la investigación. A Roberto Bustamante por sus críticas y ayuda con los borradores iniciales de algunos gráficos. A Marcelo Saco por su espontaneo apoyo a nuestro proyecto de investigación. A Mabel Cruz Rivas del INC-Huánuco por las facilidades para el acceso al sitio y desarrollo de la investigación. A Gerald Zubiaga por las coordinaciones e información proporcionada. Al Arq. Hilder Muñoz, Alcalde de la Provincia de Dos de Mayo y autoridades de La Unión, por las facilidades prestadas. Al Sr. Marcos Tiburcio Espinoza y familia por el apoyo logístico. A Cesar Guerra, investigador de la Facultad de Física de la PUCP, de modo especial por su ayuda permanente y asesoría físicomatemática. Y a Norma Matos por sus sugerencias y constante apoyo

\section{Bibliografía}

Aveni, Anthony

1993 Ancient Astronomers. St. Remy Press and Smithsonian Institution, Washington D.C.

2001 Skywatchers. A Revised and Updated Version of Skywatchers of Ancient Mexico. University of Texas Press, Austin.

Bauer Brian y David Dearborn
1998 Astronomía e Imperio en los Andes. Cuzco: Centro de Estudios Regionales Andinos "Bartolomé de las Casas".

Betanzos, Juan de

1987 [1551]Suma un narración de los Incas.

Transcripción y notas de María del

Carmen Martín Rubio. Madrid:

Ediciones Atlas.

Dearborn, David Matthew Seddo y Brian Bauer

1998 "The Sanctuary of Titicaca: Where the

Sun Returns To Earth". Latin American

Antiquity, 9(3), pp. 240-258.

Dearborn, David, Katharina Schreiber y Raymon White

1987 Intimachay: A December Solstice Observatory at Machu Picchu, Peru. American Antiquity. 52(2), pp. 346-352.

Guaman Poma de Ayala

1980 [1615] El primer nueva corónica y buen gobierno. J. Murra, R. Adorno y J. Urioste. México: Eitorial Siglo Veintiuno.

Harth-Terre, E.

1964 "El Pueblo de Huánuco Viejo". Arquitecto Peruano, 320-21, pp.1-20. Lima.

Hyslop, John

1985 Inkawasi, the New Cuzco: Cañete, Lunahuaná, Perú. Institute of Andean Research, New York; BAR, Oxford.

1990 Inka Settlement Planning. University of Texas Press, Austin.

Matos, Ramiro

1986 El Ushnu de Pumpu. Cuicuilco NŸ8:4561, México: INAH.

1994 Pumpu: Centro Administrativo Inka de la Puna de Junín. Lima: Editorial Horizonte.

Meeus, Jean

2000a Astronomical Algorithms. Williann-Bell, Inc. Richmond, Virginia.

200b Mathematical Astronomy Morsels. Williann-Bell, Inc. Richmond, Virginia.

Morris, C.

1987 "Arquitectura y Estructura del Espacio en Huanuco Pampa". Cuadernos 12: 27 - 
45. Buenos Aires: Instituto Nacional de Antropología,

Morris, C. y D. Thompson

1970 "Huánuco Viejo: An Inca Administrative Center". American Antiquity, vol. 35, NŸ3:344-362.

1985 Huánuco Pampa: An Inca City and its Hinterland. Thames and Hudson, London.

Murra, John

1978 La organización económica del Estado Inca. Siglo XXI editores. México.

Pino, José Luis

2001a "La idea de Ushnu en los Andes Centrales”. Ponencia presentada en XIII Congreso Peruano del hombre y la cultura Andina. Lima.

2001b "El Ushnu Inka: ritual, poder y orden astronómico en la Sierra Central del Chinchaysuyu". Ponencia presentada en XIII Congreso Peruano del Hombre y la Cultura Andina. Lima.

2003 El Ushnu Inka y la organización de espacio en los principales tampus de los Wamanis de la Sierra Central del Chinchaysuyo. Ponencia presentada en el

51 Congreso Internacional de Americanistas, Symposium ARQ-12, Santiago de Chile.

Shea, D.

1966 El Conjunto Arquitectónico Central en la Plaza de Huánuco Viejo. Cuadernos de Investigación, NŸ1: 108-116. Huánuco: UNHV.

Taylor, Gerald

1999 Ritos y tradiciones de Huarochirí. Lima: IFEA.

2000 Camac, camay y camasca, y otros ensayos sobre Huarochirí y Yauyos. IFEA. Lima.

Urton, Gary

1981 At the Crossroads of the Earth and the Sky: An Andean Cosmology. University of Texas Press, Austin.

1985 "La Orientación en la Astronomía Quechua e Inca", en La Tecnología en el
Mundo Andino, editado por H. Letchman y A. M. Soldi., pp. 475-490. México: UNAM.

2001 A calendrical and Demographic tomp text from Northern Peru. Latin American Antiquity, 12(2), pp. 127-147.

Wachtel, Nathan

1973 Sociedad e ideología, ensayos de Historia Antropológica Andinas. Lima: IEP.

Ziolkowski, Mariuz y Robert Sadowski

1992 La Arqueoastronomía en la Investigación de las Culturas Andinas. Colección Pendoneros NŸ9, Quito: Banco Central del Ecuador.

Zuidema, R. Tom

1974/76 La imagen del Sol y la Huaca de Susurpuquio en el sistema astronómico de los Incas en el Cuzco". Journal de la Société des Américanistes NŸG3: 199. 230.

1977 The Inca Calendar. En Native American Astronomy, Anthony Aveni editor, pp 219-259. University of Texas Press, Austin.

1980 "El Ushnu". Revista de la Universidad Complutense, vol. 28, NŸ117:317-362. Madrid.

1981 Inca Observations of the Solar and Lunar Passages Through Zenith and Anti-Zenith at Cuzco, en Archaeoastronomy in the Americas, editado por R. Williamson, pp.319-342. Ballena Press. Los Altos, California.

1983 "Catachillay: El Rol de las Pleiades y de la Cruz del Sur y Alfa y Beta Centauri en el Calendario de los Inkas." Fénix 28/29:130-150. Revista de la Biblioteca Nacional del Perú.

1989 Reyes guerreros. Ensayos de cultura andina. Fomciencias. Lima.

Zuidema, R. Tom y Gary Urton

1976 La Constelación de la Llama en los Andes Peruanos. Allpanchis Phuturinqa 9:59-119. Cuzco. 